\title{
Representation of Salty Taste Stimulus Concentrations in the Primary Gustatory Area in Humans
}

\author{
Tatsu Kobayakawa - Sachiko Saito • Naomi Gotow • \\ Hisashi Ogawa
}

Received: 22 October 2007 / Accepted: 31 July 2008 / Published online: 5 September 2008

(C) The Author(s) 2008. This article is published with open access at Springerlink.com

\begin{abstract}
The relationship between neuronal activity in the human gustatory cortex and the concentrations of taste stimuli is not well understood. In this study, we recorded changes in the magnetic fields of the human brain in response to four concentrations of $\mathrm{NaCl}(30 \mathrm{mM}, 100 \mathrm{mM}$, $300 \mathrm{mM}$, and $1 \mathrm{M}$ ) and measured the magnitude and shortest latency of the equivalent current dipole (ECD) in the primary gustatory area of the cerebral cortex (PGA). The average magnitude of ECDs in the PGA increased in a concentration-dependent manner throughout the entire range of $\mathrm{NaCl}$ concentrations. The shortest latency of the ECD, however, did not vary with the stimulus concentration.
\end{abstract}

Electronic supplementary material The online version of this article (doi:10.1007/s12078-008-9030-4) contains supplementary material, which is available to authorized users.

T. Kobayakawa $(\square) \cdot$ N. Gotow

Multimodal Integration Research Group,

Institute for Human Science and Biomedical Engineering,

National Institute of Advanced Industrial

Science and Technology (AIST),

Higashi 1-1-1,

Tsukuba, Ibaraki 305-8566, Japan

e-mail: kobayakawa-tatsu@aist.go.jp

N. Gotow

e-mail: nao-gotow@aist.go.jp

S. Saito

Saito Sachiko Taste and Smell Institute,

Shimohirooka 410-151,

Tsukuba, Ibaraki 305-0042, Japan

e-mail: am104483@mail1.accsnet.ne.jp

\section{H. Ogawa}

Department of Neurology, Kumamoto Kinoh Hospital,

Yamamuro 6-8-1,

Kumamoto 860-8518, Japan

e-mail: ogawa@juryo.or.jp
Keywords Concentration · Human · Latency · Magnetoencephalography (MEG) · Magnitude . Primary Gustatory Area

\section{Introduction}

Evaluation of stimulus intensity is a critical aspect of human perception. Several psychophysical studies have related taste stimulus concentrations to subsequent human responses, such as intensity perception and reaction time (i.e., the time between presentation of the taste stimulus and a response, e.g., pressing a hand button immediately after perception; Beebe-Center and Waddell 1948; Yamamoto and Kawamura 1981; Halpern 1986). The means by which taste stimulus concentrations are encoded in the primary gustatory area, however, could not been determined solely by psychophysical studies. Consequently, we have yet to fully understand how human gustatory cortices represent signal intensity in response to various concentrations of tastants and how these activation latencies are related to reaction times.

Recent advances in brain imaging techniques, on the other hand, have made it possible to nonintrusively observe neural activity within the living human brain. The use of techniques such as functional magnetic resonance imaging (fMRI), positron emission tomography (PET), and magnetoencephalography (MEG) has begun to elucidate the relationships between human gustatory perception and brain activity (Kobayakawa et al. 1996, 1999; Small et al. 1999, 2003; Faurion et al. 1999; Cerf-Ducastel et al. 2001; Mizoguchi et al. 2002). Among these methods, MEG is superior to other methods in temporal resolution, and equivalent to those in spatial one, unless several regions are activated simultaneously. 
We have previously measured gustatory-evoked magnetic fields in humans. These studies located the primary gustatory area (PGA) both at the transition between the parietal operculum and insula and at the bottom of the central sulcus because these regions displayed the shortest recorded latencies (Kobayakawa et al. 1996, 1999; Mizoguchi et al. 2002). The former area roughly corresponds to area $G$ in non-human primates (Sanides 1968; Ogawa 1994), although it differs in position along the anterior-posterior axis of the brain. We have therefore named this region area $G$ in humans. The activation of other cortical areas, including the frontal operculum and the superior temporal sulcus, was also observed a few hundred milliseconds after the initial activation in human area G (Kobayakawa et al. 1999).

In the present study, we aimed to investigate the manner in which human area $G$ encodes the concentration of a taste stimulus, by measuring the magnitude and the shortest latency of the equivalent current dipoles (ECDs) estimated in response to various stimulus concentrations in area $\mathrm{G}$.

\section{Materials and Methods}

\section{Participants}

Eight healthy volunteers (four women and four men), aged 22 to 41 years (mean age, $27.6 \pm 5.8$ years old), participated in the experiment. This study was conducted in accordance with the revised version of the Helsinki declaration. All of the procedures in this study were approved by the review board in the National Institute of Advanced Industrial Science and Technology, Japan.

\section{Stimuli}

Four concentrations ( $30 \mathrm{mM}, 100 \mathrm{mM}, 300 \mathrm{mM}$, and $1 \mathrm{M}$ ) of $\mathrm{NaCl}$ desolved in deionized water were used as taste stimuli. Two volunteers did not participate in experiments using the $30 \mathrm{mM} \mathrm{NaCl}$ solution. One of these two participants was not able to distinguish between the $30 \mathrm{mM} \mathrm{NaCl}$ solution and deionized water, and the other did not participate in the experiment. All of the other participants were able to distinguish all of the salt solutions from water in all the recording sessions. All experiments, therefore, were performed under supra-threshold conditions. Of the eight participants, two did not participate in the $30 \mathrm{mM} \mathrm{NaCl}$ experiment but did participate in trials at the other concentrations. We also asked participants about the hedonic value after each session using VAS. On this scale, "very pleasant" was defined as a value of 3 , and "very aversive" was defined as -3 . The average hedonic value was $-0.17 \pm 0.06$, which was almost neutral.

\section{Procedure}

During a session, each participant underwent 40 trials in which a single $\mathrm{NaCl}$ concentration was presented. A participant was administered each of the four salt solutions over four sessions in a randomized order. In each trial, the stimulus was presented for $400 \mathrm{~ms}$ with an inter-stimulus interval of $30 \mathrm{~s}$. For chemical stimulation, a taste stimulator delivered tastants without a mechanical or thermal component at the participant's tongue (see Kobayakawa et al. 1996 for details). Taste solutions were administered in constantly flowing deionized water to negate any tactile sensation (Kobayakawa et al. 1996, 1999) over a 7-× $2.8-\mathrm{mm}$ region on the tip of the tongue where 20 to 30 taste buds have been estimated to be present in most individuals (Miller 1986). The short durations (400 ms) of stimulus presentation were achieved with a small amount of air separating the stimulants from the plain water. The area of the stimulus site was approximately the same size as the filter-paper disk that has been used to clinically evaluate gustatory function in earlier work (Tomita et al. 1986). In each session, participants were asked to place the same region of their tongues into the aperture of the Teflon tube through which fluid flowed. The temperature of the taste solutions and deionized water were maintained at $36{ }^{\circ} \mathrm{C}$ to match that of the stimulated region of the tongue. To avoid fatigue, participants were only subjected to one or two sessions per day. When the quality of MEG data was not sufficient for analysis, the session was repeated.

\section{Recording of Magnetic Fields}

A 64-channel whole-head SQUID system (CTF Systems Inc., Canada) was used to measure the gustatory-evoked magnetic fields, using a sampling rate of $250 \mathrm{~Hz}$ with a $40-\mathrm{Hz}$ low-pass filter. The location of the sensors with respect to the head was determined by measuring the magnetic fields produced by small currents delivered to three coils attached to the scalp at the nasion and two preauricular points.

\section{MEG Data Analysis}

Trials contaminated by eye movements were rejected; the remaining trials were used for calculations. Sessions with fewer than 30 successfully recorded trials were rejected. Approximately 35 trials per session per participant were used for averaging. Anatomical 3-D T1 MRI was obtained for all subjects (Siemens, 1.0 T). For source modeling, MRI head-shape data were used to determine the fitting sphere for each subject.

At the time of acquisition of MRI data, oil-filled pellets were attached to the physical landmarks used in the MEG 
experiment. The positional information given by the images of the oily pellets was used to align the MEG data with the participant's MRI.

In our estimation of ECDs, we attempted to minimize error by using Grynszpan-Geselowitz's equation (Grynszpan and Geselowitz 1973; see Kobayakawa et al. 1996). The error, $E$, was calculated according to the following formula:

$$
E=\frac{\sum_{n=1}^{64}\left(\hat{X}_{n}-X_{n}\right)^{2}}{\sum_{n=1}^{64} X_{n}^{2}}
$$

where $X_{n}$ is the observed magnitude of the neuro-magnetic field at the $n$th sensor and $\hat{X}_{n}$ is the calculated magnitude of the neuro-magnetic field at the same sensor based on the theoretical model. The goodness of fit was calculated as $(1-E)$. The locations and the magnitudes of the ECDs in each subject were estimated from the magnetic fields obtained at 64 sensor positions.

The coordinates of the dipole's centers of gravity were overlaid on individual MRI slices to identify the corresponding locations in the brain after alignment. Estimations of ECDs with high validity were performed with $>80 \%$ goodness of fit. We utilized data only when the ECDs were stable for more than $30 \mathrm{~ms}$ and maintained a suitable power of less than $100 \mathrm{nAm}$. In addition, the analyzed ECDs were located on the gray matter in MRI images. The cortical areas in which the ECDs were located were denominated using 3 -D views (axial, coronal, and sagittal). The cortical structures where ECDs were located were identified according to an atlas of the human brain (Mai et al. 1997).

We first identified the time point at which magnetic fields exhibited a local maximum in the shortest latency after the stimulus onset. By calculating the ECDs at every sampling point $(4 \mathrm{~ms})$ around this time point, we were able to determine the latency between the onset of the stimulus and the point at which the ECD had the greatest magnitude.

In cases in which two or more experimental sessions were performed with a single participant using a single $\mathrm{NaCl}$ concentration, we used average values of the ECD magnitudes and latencies for data analysis. The coordinates of estimated ECDs were transformed into those of the MNI standard brain to investigate positional changes possibly resulting from variations in $\mathrm{NaCl}$ concentration.

\section{Results}

Location of the Shortest ECD

A total of 63 experimental sessions were available for data analysis. Of the areas that responded with the shortest latencies following stimulus presentation, area $G$ (at the transition between parietal operculum and insula) was exclusively activated in 39 sessions $(62 \%)$, the central sulcus was exclusively activated in one session (1.6\%), and both area $G$ and the central sulcus were activated in 17 sessions $(27 \%)$. The total frequency of activation in area $G$ and/or the central sulcus was $90 \%$, in agreement with previous results (Kobayakawa et al. 1999). Table 1 shows the frequencies of activation in area $G$ and the central sulcus following the presentation of each of the four tastestimulus concentrations. Because we obtained only a few cases of central sulcus activation but found activations in area $\mathrm{G}$ for all the four $\mathrm{NaCl}$ concentrations, in this study, we only precisely analyzed data from area G.

Each of the graphs in Fig. 1 illustrates the superimposed magnetic fields from 64 channels for each stimulus concentration, labeled on the right. In the figure, black vertical lines show the stimulus onset, whereas gray lines indicate the point at which the ECDs were estimated because the magnetic field had clearly changed. Figure 2 shows variation in shortest activities in area $G$ in response to the four $\mathrm{NaCl}$ concentrations in a representative participant. The central row in the figure shows contour maps of the measured magnetic fields, whereas the upper and lower rows represent the calculated ECDs in the left hemisphere and in the right hemisphere overlaid on anatomical MR images, respectively. Three higher $\mathrm{NaCl}$ concentrations (100 $\mathrm{mM}$ and $1 \mathrm{M}$ ) gave rise to ECDs near the insular cortex, whereas the lowest $\mathrm{NaCl}$ concentration $(30 \mathrm{mM})$ induced an ECD at a more lateral position. One-factor analysis of variance (ANOVA) revealed no significant differences in the MNI coordinates of ECDs elicited by the four different $\mathrm{NaCl}$

Table 1 Percentage of sessions in which activations in human area $\mathrm{G}$ and the central sulcus were observed at each of the four concentrations of $\mathrm{NaCl}$

\begin{tabular}{|c|c|c|c|c|}
\hline Human area G only & $6(60 \%)$ & $15(79 \%)$ & $8(42 \%)$ & $10(67 \%)$ \\
\hline Central sulcus only & $0(0 \%)$ & $1(5.3 \%)$ & $0(0 \%)$ & $0(0 \%)$ \\
\hline Both human area $G$ and central sulcus & $3(30 \%)$ & $3(16 \%)$ & $8(42 \%)$ & $3(20 \%)$ \\
\hline
\end{tabular}




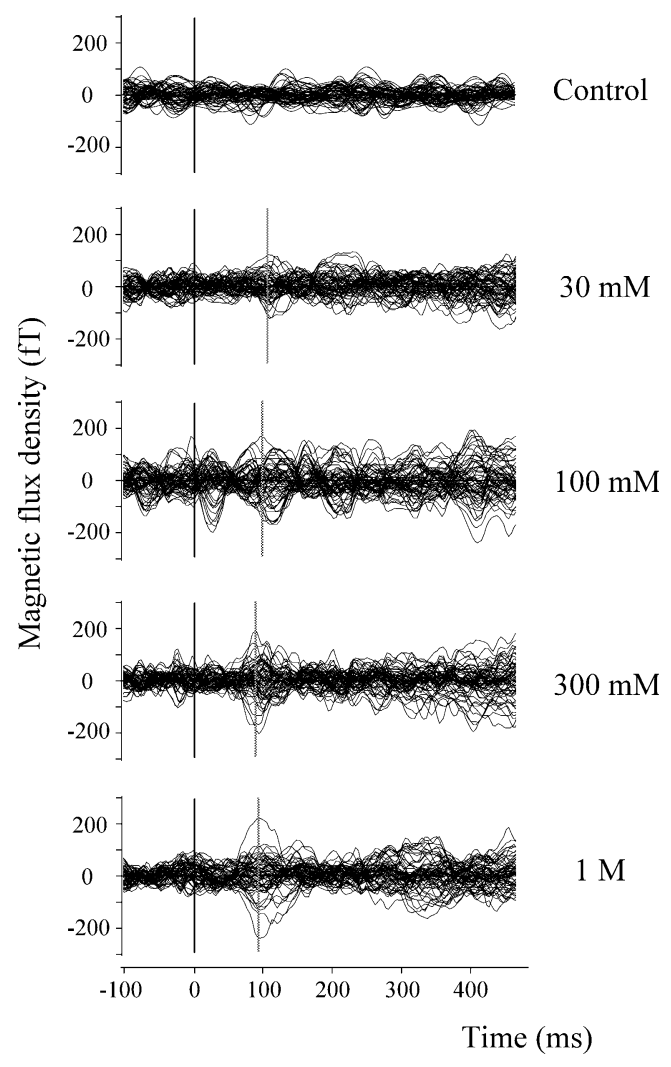

Fig. 1 The superimposed magnetic fields of 64 channels recorded in a single participant in response to four concentrations of $\mathrm{NaCl}$ and water. The concentration of $\mathrm{NaCl}$ is indicated on the right. Control represents the magnetic fields induced by deionized water. The time 0 (black lines) on the abscissa indicates the stimulus onset. Gray lines indicate the time points for the shortest peak or trough of the changes in the magnetic fields after the stimulus onset concentrations [left hemisphere $x ; F(3,24)=2.11$, not significant or n.s.: $y ; F(3,24)=1.27$, n.s.: $z ; F(3,24)=0.21$, n.s.: right hemisphere $x ; F(3,20)=1.10$, n.s.: $y ; F(3,20)=0.97$, n.s.: $z ; F(3,20)=0.12$, n.s.].

Magnitude of the Shortest ECDs

Figure 3 shows all averaged ECD amplitudes for four concentrations (eight participants for $1 \mathrm{M}, 300 \mathrm{mM}$, and $100 \mathrm{mM}$; six for $30 \mathrm{mM}$ ). Spearman's correlation coefficient between $\mathrm{NaCl}$ concentrations and ECD amplitudes was $0.69(p<0.01, n=30)$. Regression analysis yielded a regression coefficient of 15.4 , a value significantly larger than $0(p<0.01, n=30$, Student $t$ test $)$.

We analyzed ECD magnitudes within area $G$ in two different data sets, using one-factor repeated measures ANOVA: one data set consisting of the responses of the eight participants to three $\mathrm{NaCl}$ concentrations (data set $\mathrm{A}$ ) and a second data set consisting of the responses of six participants to four $\mathrm{NaCl}$ concentrations (data set $\mathrm{B}$ ). Figure 4 shows the relationship between $\mathrm{NaCl}$ concentration and the magnitude of the ECDs (filled circles) and between $\mathrm{NaCl}$ concentration and ECD latencies (filled squares) for data sets A and B. One-factor repeated measures ANOVA was applied to the magnitudes of ECDs in the two data sets. In data set A, ECD amplitude varied significantly with $\mathrm{NaCl}$ concentration $[F(2,14)=4.5, p<0.05]$. Post-hoc analyses (Tukey HSD test) indicated a significant difference in ECDs between $100 \mathrm{mM}$ and $1 \mathrm{M} \mathrm{NaCl}(p<0.05)$. ECD amplitude also varied significantly with $\mathrm{NaCl}$ concentration in data set $\mathrm{B}[F$

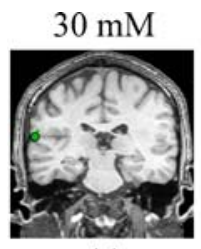

4.1
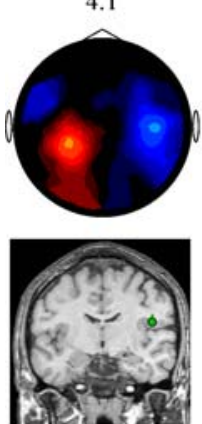

4.8

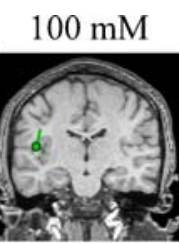

25.1
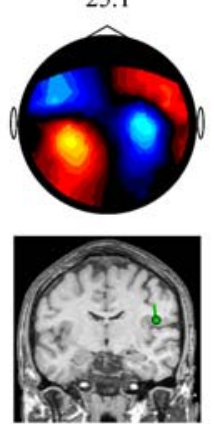

24.6

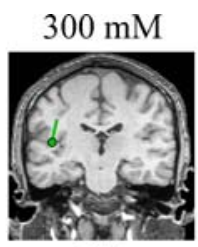

35.4
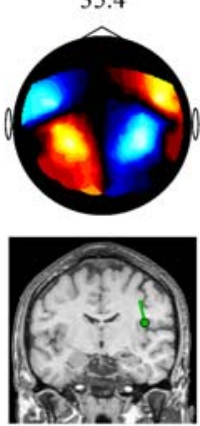

38.3
$1 \mathrm{M}$

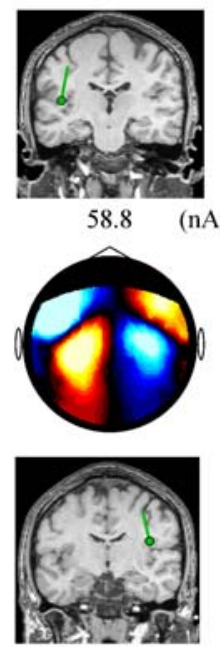

$51.4 \quad(\mathrm{nAm})$

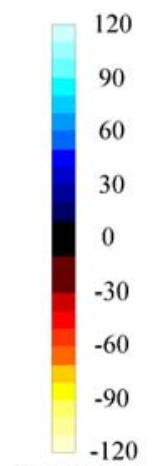

Magnetic flux

density (fT)

Fig. 2 The shortest cortical activities recorded in response to four $\mathrm{NaCl}$ concentrations, in a single participant. The central row shows contour maps of the magnetic fields recorded. The color scale used to depict the density of magnetic flux is indicated on the right. All isocontour maps show that the magnetic field was directed from the left-front area of the head to the left-back and from the right-back to

the right-front. The upper and lower rows illustrate ECDs (green dots) with the shortest latency relative to stimulus onset in the left and right hemispheres, respectively, overlaid on anatomical MR images. Numerals underneath the anatomical images indicate the ECD magnitudes (nAm), which are represented by the lengths of the green lines in the images 


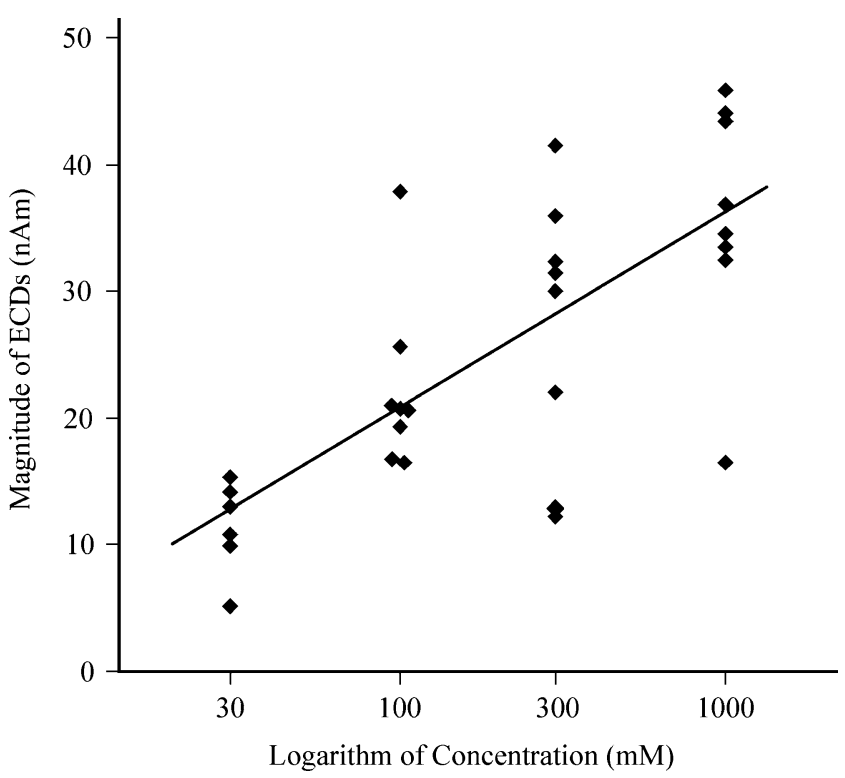

Fig. 3 Scatter plot of averaged ECD amplitudes for each participant against $\mathrm{NaCl}$ concentrations. A regression coefficient was 15.4, significantly greater than 0 , and Spareman's correlation coefficient between concentrations and ECD amplitudes was 0.78

$(3,15)=7.93, p<0.01]$. Post-hoc analysis (Tukey HSD test) indicated significant differences in ECDs between $30 \mathrm{mM}$ and $1 \mathrm{M}(p<0.01), 30 \mathrm{mM}$ and $300 \mathrm{mM}(p<0.05)$, and $30 \mathrm{mM}$ and $100 \mathrm{mM}(p<0.05)$.

\section{Latencies of the Shortest ECDs}

One-factor repeated measures ANOVA did not detect a significant difference in the shortest latency of area $G$ activation across the $\mathrm{NaCl}$ concentrations in either data set
A $[F(2,14)=1.6$, n.s. $]$ or data set $\mathrm{B}[F(3,15)=0.97$, n.s. $]$ (squares in Fig. 4).

\section{Discussion}

\section{Location of the Human PGA}

Non-invasive brain imaging has been applied to the localization of the primary and higher order gustatory cortices in human beings. Studies using fMRI or PET have identified several cortical regions that are activated by gustatory stimulation (Cerf-Ducastel et al. 2001; Small et al. 2003). Moreover, the frontal operculum, topographically and cytoarchitectonically corresponding to the PGA in subhuman primates (Sanides 1968; Pritchard et al. 1986; Ogawa 1994), has been proposed as the PGA (Small et al. 1999, 2003; O'Doherty et al. 2001), although it has not been confirmed whether this region was activated with the shortest latency among all the cortices concerned.

MEG, on the other hand, another non-invasive brain imaging technique with superior temporal resolution, revealed that the cortical activity with the shortest latency after the onset of taste stimulation with $1 \mathrm{M} \mathrm{NaCl}$ and $3 \mathrm{mM}$ saccharin occurs at the transition between the parietal operculum and insula (human area $\mathrm{G}$ ) and at the ventral end of the central sulcus (Kobayakawa et al. 1999). This location is consistent with previous clinical findings. These locations are the parietal operculum and the dorsal posterior insula. Penfield and coworkers $(1954,1955)$ elicited taste sensation by electrical stimulation of these areas during brain surgery; also, hematomas in the posterior central
(A)

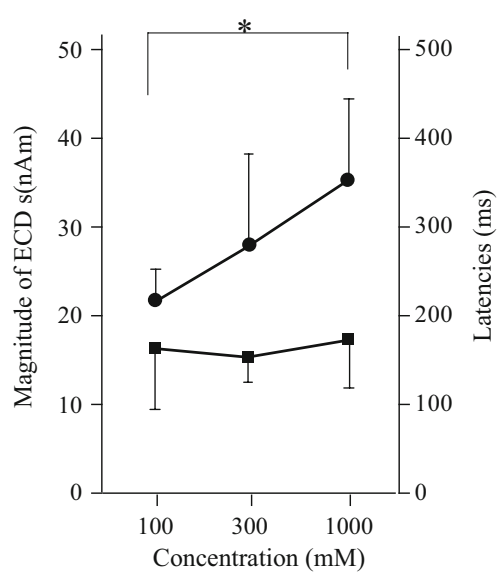

Fig. 4 a, b The relationships between the stimulus concentration and the averaged ECD magnitude or peak latencies of the ECDs for data sets $\mathrm{A}$ and B. Filled circles and squares indicate the averaged magnitudes of ECDs and latencies of the ECDs, respectively. The error bar indicates the standard error. One-factor repeated measures
(B)

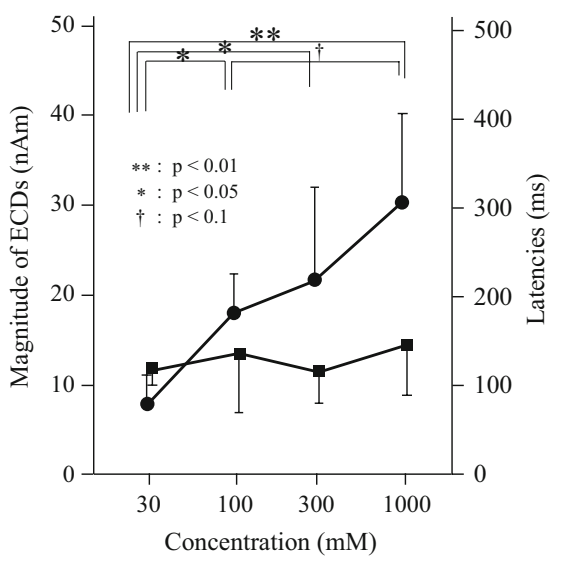

ANOVA revealed significant differences in the ECD magnitudes evoked by the different $\mathrm{NaCl}$ concentrations in data sets $\mathrm{A}(p<0.05)$ and $\mathrm{B}(p<0.01)$ but no significant difference in the latencies after the stimulus onset in either data set. ${ }^{\dagger} P<0.1 ; * P<0.05$; $* * P<0.01$; (Posthoc analyses, Tukey HSD test) 
gyrus were associated with dysgeusia in three gunshot patients (Börnstein 1940).

Using fMRI, Ogawa et al. (2005) first revealed tasteinduced activations in the parietal operculum and posterior insula by repeated presentations of a tastant pulse during a stimulation period, the same means used to activate the primary visual or auditory cortices. Quite recently, fMRI studies reported by Nitschke et al. (2006) and Veldhuizen et al. (2007) also revealed taste-induced activities in the parietal operculum and posterior insula as well as in the frontal operculum. The former studies, however, were ambiguous about the precise location of the PGA. In support of the PGA's residence in the frontal operculum, Veldhuizen et al. (2007) ascribed the activation in the parietal operculum to activities in oral tactile afferents. On the other hand, in support of our MEG findings (Kobayakawa et al. 1996, 1999), Komiyama et al. (2007) found no activation by $3 \mathrm{M} \mathrm{NaCl}$ (aversive high concentration) in either the parietal operculum or the posterior insula in patients who had chorda tympani nerves bilaterally resected during middle ear surgeries. They also found that mechanical stimulation at the tip of the tongue evoked the shortest activations only at the oral cavity representation region in the central sulcus (probably its posterior wall), not at the parietal operculum. These results clarified that the taste-induced activities of the parietal operculum and posterior insula revealed by MEG are carried by taste afferents but not by the oral tactile afferents.

In the present study, therefore, we chose the human area $\mathrm{G}$, consisting of the parietal operculum and posterior insula, to investigate the representation of taste stimulus intensity in the PGA.

\section{Information Coding of Stimulus Concentration}

Our findings showed that, in human area $G$, the activity with the shortest latency after the stimulus onset increased with stimulus concentration. Furthermore, significant changes in ECD amplitudes were consistently observed as the tastant concentration varied more than tenfold.

In some sensory modalities, such as auditory or tactile sensation, a close relationship has been consistently observed between cortical activity in the primary sensory area and the physical intensity of stimulus. Bak et al. (1985) reported that the peak magnetic-field amplitude $100 \mathrm{~ms}$ after the stimulus onset correlated well with the decibel level of the sound stimulus. Similarly, the amplitude of magnetic-field changes within the primary somatosensory area can be enhanced by increasing the intensity of electrical stimuli applied to the skin (Jousmäki and Forss 1998; Torquati et al. 2002). The trends observed for these sensory modalities are in good agreement with our present findings for gustation.

Perceived intensity may not always be consistent with the amplitude of the first activation in the PGA because the process of intensity perception includes neural processes at many higher levels, including memory access. The mechanism of processing intensity perception after PGA is still unclear; hopefully, further study will clarify this issue in the near future.

In an fMRI study using two different concentrations of tastants, Small et al. (2003) reported that the magnitude of activation in the frontal operculum and the anterior part of the insula increased with tastant concentration. Although, based on these results, they maintained that the frontal operculum and anterior insula were the PGA, our MEG studies (Kobayakawa et al. 1996, 1999) had already revealed that the frontal operculum and anterior insula were activated a few hundred milliseconds after area $G$ and the central sulcus.

Nitschke et al. (2006) reported that PGA activation is modulated by participants' expectation. Kobayakawa et al. (1999) reported repeated activation of area G after the shortest latency. To reconcile these observations, we suggest that the earliest activation of area $G$ encodes the objective nature of taste, while later activations of area $G$ may be modified by the expectation to the properties of upcoming tastants.

In order to functionally differentiate the PGA from higher order gustatory cortices in sub-human primates, Ifuku et al. (2003) recorded neuronal activities from gustatory cortices of a Japanese macaque engaged in a $\mathrm{NaCl}$-water discrimination delayed GO/NOGO task. They reported that almost all neurons in the PGA (area G and the oral part of area 3) maintained constant response patterns to a given tastant, independent of experimental conditions (gustatory naturedependent response). On the contrary, most neurons in higher order gustatory cortices (the precentral operculum and orbitofrontal cortex) changed their response patterns depending upon the consequent behavioral task. Thus, in subhuman primates, higher order gustatory cortices may be more easily modulated by psychological factors, such as behavioral context or expectation, compared to the PGA. In order to reveal the potential modulation by psychological factors, however, detailed study in the human PGA will be required.

Temporal Aspects of Cortical Activity and Stimulus Concentration

The average latency of the shortest activation in human area $\mathrm{G}$ after the stimulus onset was $160 \mathrm{~ms}$, consistent with the result of Kobayakawa et al. (1999), but this is $34 \mathrm{~ms}$ longer than the value reported in Mizoguchi et al. (2002). In the present study and that of Kobayakawa et al. (1999), the longer estimated latency can probably be ascribed to the usage of only single optical sensor placed before the stimulation site to detect the arrival of the tastant and the 
estimation on the basis of the flow rate of the time at which the stimulus reaches the stimulation site. On the other hand, Mizoguchi et al. (2002) used two sensors located before and after the stimulation site and precisely calculated the time at which the tastant arrived at the stimulation site, based on the time difference of the signal outputs between the two sensors, instead of the flow rate.

Yamamoto and Kawamura (1981) reported that the gustatory response time in humans decreases with increasing tastant concentration from $940 \mathrm{~ms}$ for $40 \mathrm{mM} \mathrm{NaCl}$ to $400 \mathrm{~ms}$ for $1 \mathrm{M} \mathrm{NaCl}$. Halpern (1986) and Saito et al. (1998) observed similar changes in reaction times with increases in $\mathrm{NaCl}$ concentrations. In the present experiment, however, shortest latency of the activity in area $\mathrm{G}$ did not significantly change with changes in $\mathrm{NaCl}$ concentrations.

Torquati et al. (2002) and Bak et al. (1985) found that the onset latencies of cortical activity did not vary with changing intensities of supra-threshold stimuli. In the present study, the shortest activation in human area $\mathrm{G}$ did not exhibit any significant changes in the onset latency with varying stimulus concentrations, consistent with findings in other sensory areas and supra-threshold stimulus intensities.

Ito et al. (2001) also noted that reaction time decreased with increases in tastant concentration in subhuman primates; however, the onset latencies of neuronal responses in the PGA (area 3, area $\mathrm{G}$ and other cortical areas) were not affected by changes in $\mathrm{NaCl}$ concentrations. Scott et al. (1991) also noted that, in the macaque, the activity of single cortical neurons increased response magnitudes with increasing tastant concentration, whereas the onset latency for these neurons did not change significantly. The present findings detailing the relationship between $\mathrm{NaCl}$ concentrations and the response magnitudes and latencies of the neuronal activity in human area $\mathrm{G}$ are consistent with the results obtained from the PGA in monkeys. Taken together, it is indicated that area $\mathrm{G}$ does not contribute to concentration-dependent changes in the reaction time.

Higher gustatory cortices may yield concentrationdependent changes in onset latencies, corresponding to changes in a subject's reaction time. Such cortices may alter response magnitudes depending upon not only the concentration of the stimulus but also on other aspects of gustatory perception. The mechanisms by which these higher gustatory cortices encode gustatory information will be a topic for future studies.

\section{Conclusion}

In the present study, we investigated the manner in which human PGA encodes the concentration of a taste stimulus by measuring the magnitude and the shortest latency of the ECDs for various stimulus concentrations. The average magnitude of ECDs in the PGA increased in a concentration-dependent manner throughout the entire range of $\mathrm{NaCl}$ concentrations studied. The shortest latency of the ECD, however, did not vary with variations in the stimulus concentration.

Acknowledgments This work was partly supported by a grant-in-aid from the Ministry of Education, Culture, Sports, Science and Technology of Japan (no. 14390059 to S.S. and group) and also supported by a grant from Program for Promotion of Basic Research Activities for Innovative Biosciences (PROBRAIN).

Open Access This article is distributed under the terms of the Creative Commons Attribution Noncommercial License which permits any noncommercial use, distribution, and reproduction in any medium, provided the original author(s) and source are credited.

\section{References}

Bak C-K, Lebech J, Saermark K (1985) Dependence of the auditory evoked magnetic field (100 msec signal) of the human brain on the intensity of the stimulus. Electroencephalogr Clin Neurophysiol 61(2):141-149

Beebe-Center J-C, Waddell D (1948) A general psychological scale of taste. J Psychol 26:517-524

Börnstein W-S (1940) Cortical representation of taste in man and monkey. II. The localization of the cortical taste area in man and a method of measuring impairment of taste in man. Yale J Biol Med 13:133-156

Cerf-Ducastel B, Van de Moortele P-F, MacLeod P, Le Bihan D, Faurion A (2001) Interaction of gustatory and lingual somatosensory perceptions at the cortical level in the human: a functional magnetic resonance imaging study. Chem Senses 26 (4):371-383

Faurion A, Cerf B, Van de Moortele P-F, Lobel E, Mac Leod P, Le Bihan D (1999) Human taste cortical areas studied with functional magnetic resonance imaging: evidence of functional lateralization related to handedness. Neurosci Lett 277(3):189192

Grynszpan F, Geselowitz D-B (1973) Model studies of the magnetocardiogram. Biophys J 13(9):911-925

Halpern B-P (1986) Constraints imposed on taste physiology by human taste reaction time data. Neurosci Biobehav Rev 10 (2):135-151

Ifuku H, Hirota S, Nakamura T, Ogawa H (2003) Neuronal activities in the monkey primary and higher order gustatory cortices during a taste discrimination delayed GO/NOGO task and after reversal. Neurosci Res 47(2):161-175

Ito S, Ohgushi M, Ifuku H, Ogawa H (2001) Neuronal activity in the monkey fronto-opercular and adjacent insular/prefrontal cortices during a taste discrimination $\mathrm{GO} / \mathrm{NOGO}$ task: response to cues. Neurosci Res 41(3):257-266

Jousmäki V, Forss N (1998) Effects of stimulus intensity on signals from human somatosensory cortices. Neuroreport 9(15):34273431

Kobayakawa T, Endo H, Ayabe-Kanamura S, Kumagai T, Yamaguchi Y, Kikuchi Y, Takeda T, Saito S, Ogawa H (1996) The primary gustatory area in human cerebral cortex studied by magnetoencephalography. Neurosci Lett 212(3):155-158

Kobayakawa T, Ogawa H, Kaneda H, Ayabe-Kanamura S, Endo H, Saito S (1999) Spatio-temporal analysis of cortical activity 
evoked by gustatory stimulation in humans. Chem Senses 24 (2):201-209

Komiyama A, Kobayakawa T, Toda H, Gotow N, Ikeda M, Saito S (2007) A high-concentration $\mathrm{NaCl}$ solution does not stimulate the human trigeminal nerve at the tip of the tongue. Acta Oto-laryngol 127(7):754-759

Mai J-K, Assheuer J, Paxinos G (1997) Atlas of the human brain. Academic, New York, USA

Miller J-I (1986) Variation in human fungiform taste bud densities among regions and subjects. Anat Rec 216(4):474-482

Mizoguchi C, Kobayakawa T, Saito S, Ogawa H (2002) Gustatory evoked cortical activity in humans studied by simultaneous EEG and MEG recording. Chem Senses 27(7):629-634

Murayama N, Nakazato N, Hatanaka S, Fujita S, Igasaki T, Kanno A, Yoshimoto $\mathrm{T}$ (1996) Gustatory evoked magnetic fields in humans. Neurosci Lett 210(2):121-123

Nitschke J-B, Dixon G-E, Sarinopoulos I, Short S-J, Cohen J-D, Smith E-E, Kosslyn S-M, Rose R-M, Davidson R-J (2006) Altering expectancy dampens neural response to aversive taste in primary taste cortex. Nat Neurosci 9(3):435-442

O’Doherty J, Rolls E-T, Francis S, Rowtell R, McGlone F (2001) Representation of pleasant and aversive taste in the human brain. J Neurophysiol 85(3):1315-1321

Ogawa H (1994) Gustatory cortex of primates: anatomy and physiology. Neurosci Res 20(1):1-13

Ogawa H, Wakita M, Hasegawa K, Kobayakawa T, Sakai N, Hirai T, Yamashita Y, Saito S (2005) Functional MRI detection of activation in the primary gustatory cortices in humans. Chem Senses 30(7):583-592

Penfield W, Faulk M-E (1955) The insula; further observations on its function. Brain 78(4):445-470

Penfield W, Jasper H (1954) Epilepsy and the functional anatomy of the human brain. Little, Brown and Company, Boston, USA
Pritchard T-C, Hamilton R-B, Morse J-R, Norgren R (1986) Projections of thalamic gustatory and lingual areas in the monkey, Macaca fascicularis. J Comp Neurol 244(2):213-228

Saito S, Endo H, Kobayakawa T, Ayabe-Kanamura S, Kikuchi Y, Takeda T, Ogawa H (1998) Temporal process from receptors to higher brain in taste detection studied by gustatory-evoked magnetic fields and reaction times. Ann N Y Acad Sci 855:493-497

Sanides F (1968) The architecture of the cortical taste nerve area in squirrel monkey (Saimiri sciureus) and their relationships to insular, sensorimotor and prefrontal regions. Brain Res 8(1):97124

Scott T-R, Plata-Salaman C-R, Smith V-L, Giza B-K (1991) Gustatory neural coding in the monkey cortex: stimulus intensity. J Neurophysiol 65(1):76-86

Small D-M, Zald D-H, Jones-Gotmen M, Zatorre R-J, Pardo J-V, Frey S, Petrides M (1999) Human cortical gustatory areas; a review of functional neuroimaging data. Neuroreport 10(1):7-14

Small D-M, Gregory M-D, Mak Y-E, Gitelman D, Mesulam M-M, Parrish T (2003) Dissociation of neuronal representation of intensity and affective valuation in human gustation. Neuron 39 (4):701-711

Tomita H, Ikeda M, Okuda Y (1986) Basis and practice of clinical taste examinations. Auris Nasus Larynx 13(Suppl 1):1-15

Torquati K, Pizzella V, Penna S-D, Franciotti R, Babiloni C, Rossini P-M, Romani G-L (2002) Comparison between SI and SII responses as a function of stimulus intensity. Neuroreport 13 (6):813-819

Veldhuizen M-G, Bender G, Constable R-T, Small D-M (2007) Trying to detect taste in a tasteless solution: modulation of early gustatory cortex by attention to taste. Chem Senses 32(6):569-581

Yamamoto T, Kawamura Y (1981) Gustatory reaction time in human adults. Physiol Behav 26(4):715-719 